\title{
In vitro evaluation of gel-encapsulated adipose derived stem cells: Biochemical cues for in vivo peripheral nerve repair
}

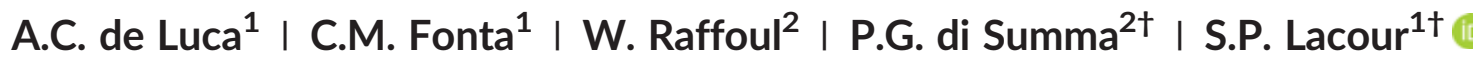

${ }^{1}$ Bertarelli Foundation Chair in Neuroprosthetic Technology, Laboratory for Soft Bioelectronic Interfaces, Institute of Microengineering, Institute of Bioengineering, Centre for Neuroprosthetics, École

Polytechnique Fédérale de Lausanne (EPFL), Lausanne, Switzerland

${ }^{2}$ Department of Plastic, Reconstructive and Hand Surgery, University Hospital of Lausanne (CHUV), Lausanne, Switzerland

\section{Correspondence}

Stéphanie P. Lacour, EPFL STI IMT|IBI LSBI Campus Biotech B3.2 Chemin des Mines 9,

$\mathrm{CH}-1202$ Geneva, Switzerland.

Email: stephanie.lacour@epfl.ch

Funding information

Swiss National Science Foundation, Grant/ Award Number: CR3212_149609

\begin{abstract}
Adipose-derived stem cells (ASC) are becoming one of the most exploited cells in peripheral nerve repair. They are fast-growing and able to protect neurons from apoptosis; they can reduce postinjury latency and the risk of muscle atrophy. This study evaluates laminin-loaded fibrin gel as an ASC-carrying scaffold for nerve repair. In vitro, ASC retained their proliferative activity but showed significant increase in proliferation rate when encapsulated in gels with low laminin concentrations (i.e., $1 \mu \mathrm{g} / \mathrm{mL}$ ). We observed a linear decrease of ASC proliferation rate with increasing laminin concentration from 1 to $100 \mu \mathrm{g} / \mathrm{mL}$. We next examined the effect of the ASC-carrying fibrin gels on in vitro dorsal root ganglia (DRG) neurite extension, then in vivo sciatic nerve regeneration in adult rats. The ASC-carrying gel was embedded in 15-mm-long, 1.5-mm-diameter polydimethylsiloxane regenerative conduits for in vivo evaluation. At 8-week post implantation, robust regeneration was observed across the long gap. Taken together, these results suggest ASC-carrying gels are a potential path to improve the efficacy of nerve regeneration through artificial guidance conduits and electrode nerve interfaces.
\end{abstract}

\section{KEYWORDS}

adipose stem cells, cell transplantation, fibrin, laminin, nerve regeneration

\section{1 | INTRODUCTION}

Stem cells have the ability to self-renew and differentiate into specific functional phenotypes (Tohill \& Terenghi, 2004), depending on their origin. Among the various sources in the body, white adipose tissue allows for harvesting a rich population of multipotent stem cells, which are able to differentiate into the Schwann cell (SC) phenotype, suggesting promising opportunities in neural engineering and regenerative medicine (Kingham et al., 2007). SC are the most important glial cells in the peripheral nervous system and are actively involved in the process of nerve regeneration after injury (Ide, 1996). However, the beneficial role of transplanted SC at the site of injury of a peripheral nerve remains limited because SC atrophy over time, and their harvesting procedure is invasive and time-consuming (Gordon, Tyreman, \& Raji, 2011; Hoke, 2006; Saheb-Al-Zamani et al., 2013). Compared with SC, adipose-derived stem cells (ASC) can be harvested from white adipose tissue with traditional, non-invasive liposuction techniques, and isolated through enzymatic and mechanical digestion procedures (Zuk et al., 2002; Kingham,

\footnotetext{
${ }^{\dagger}$ These authors contributed equally to this work and share co-last authorship.
}

Mantovani, \& Terenghi, 2011; de Luca, Faroni, \& Reid, 2015). ASC present an elevated proliferation rate thereby reducing delays for cell transplantation (Gomillion \& Burg, 2006); they prevent neuron apoptosis (Reid et al., 2011; Wei et al., 2009) and are characterized by a low immunological profile (Faroni, Smith, \& Reid, 2014). ASC have been successfully transplanted in acellular nerve grafts in rat models (Liu et al., 2011; Luo, Zhang, Zhang, \& Jin, 2012) and associated to robust nerve regeneration including remyelination (Carlson et al., 2011; Suganuma et al., 2013), reduced muscular atrophy (Santiago, Clavijo-Alvarez, Brayfield, Rubin, \& Marra, 2009) and inflammation (Marconi et al., 2012).

A critical element in cell transplantation is the delivery to a specific target tissue, allowing them to engraft and integrate within the hosting body (Seidel, Borczynska, Rozwadowska, \& Kurpisz, 2009). Cells may be directly injected at the injury site in case of a localized lesion without neural tissue disconnection. Alternatively, when axotomy occurs, a guidance conduit may be sutured between the nerve stumps, creating a confined physical environment to support regeneration (Gu, Ding, \& Williams, 2014). Biodegradable matrices can then act as fillers and provide a valid scaffold for cell transplantation, ensuring homogenous cells' distribution and accurate delivery to a targeted 
area. In this view, fibrin has been used as a delivery vehicle of growth factors and cells (Martino, Briquez, Ranga, Lutolf, \& Hubbell, 2013; Sacchi et al., 2014; Tortelli, Pisano, Briquez, Martino, \& Hubbell, 2013), as well as a scaffolding matrix for nerve tissue engineering (di Summa et al., 2011; Longo et al., 2015).

A dynamic and interactive microenvironment engineered to mimic the native tissue will influence the behaviour and fate of endogenous, recruited and transplanted cells, tailoring the regeneration and development of the injured tissue. Adhesive proteins, such as fibronectin or laminin, are major components of the extracellular matrix (ECM) and remarkably support the integration of artificial scaffolds with the host tissue (de Luca, Faroni, Downes, \& Terenghi, 2016; de Luca, Stevens et al., 2013; di Summa, Kalbermatten, Raffoul, Terenghi, \& Kingham, 2013; Gattazzo, Urciuolo, \& Bonaldo, 2014). The addition of these molecules provides in fact a rich environment for cell adhesion and migration, activating and/or inhibiting the production of biochemical factors necessary for proliferative and syntheticrin processes (Chernousov \& Carey, 2000).

With these observations in mind, we have developed a fibrin matrix, supplemented with adhesive proteins and suitable for cell transplantation. We tested the effect of the hybrid cell-gel system in vitro then in vivo against cell viability and peripheral nerve sprouting. We selected polydimethylsiloxane (PDMS) as an inert in vitro substrate and in vivo construct material (Minev, Moshayedi, Fawcett, \& Lacour, 2013, Minev et al., 2015; Srinivasan et al., 2015). Although alternative conduit materials such as biodegradable fibrin are often used to prepare regenerative nerve conduits, we opted for PDMS, a material of choice for cuff electrodes, in the prospect of subsequent regenerative nerve electrode implants. Laminin was selected given its critical role in myelin formation and axonal sorting (Tashiro et al., 1989). The interactions between dorsal root ganglia (DRG) and ASC were investigated in a three-dimensional in vitro coculture system. The ability of such hybrid cell-gel system loaded in inert PDMS conduits to support regeneration was then verified using an in vivo total axotomy sciatic nerve model in rats over 8 weeks.

\section{2 | MATERIALS AND METHODS}

\section{1 | Harvest and culture of ASC}

ASC were isolated from rats using a previously established protocol (de Luca et al., 2015), and in accordance with the local veterinary commission of the canton of Vaud (Lausanne, Switzerland).

Briefly, following euthanasia, visceral and inguinal fat was harvested, triturated, and digested with a $0.2 \% \mathrm{w} / \mathrm{v}$ collagenase type I solution (Gibco). After filtration of the cell suspension through a $100-\mu \mathrm{m}$ cell strainer, blood cells were removed with the addition of a red blood cells lysis buffer (Sigma). The remaining cells were incubated at $37{ }^{\circ} \mathrm{C}, 5 \% \mathrm{CO}_{2}$ and subcultured in growth medium composed of minimum essential medium (a-MEM, Gibco), 10\% fetal bovine serum (FBS), 200-mM L-glutamine, 1\% penicillin-streptomycin (PS).

\subsection{Influence of adhesive molecules on ASC culture in vitro}

To study the relevance of adhesive molecules on in vitro ASC cultures in contact with PDMS surfaces, laminin and fibronectin (Sigma) were coated on the top of PDMS discs (1 cm in diameter). PDMS (10:1 w:w prepolymer:cross-linker ratio, Sylgard 184 Dow Corning) was cast in a polystyrene petri dish, cured at $80{ }^{\circ} \mathrm{C}$ for $2 \mathrm{hr}$ and cut with a punch.

Each disc was sterilized in $70 \%$ ethanol under a biological cabinet prior to coating with a solution of laminin or fibronectin at the final concentration of $2 \mu \mathrm{g} / \mathrm{cm}^{2}$. Five thousand cells per square centimetres were seeded on each substrate $(n=3)$ and allowed to attach for $4 \mathrm{hr}$, prior to top up of the wells with the final volume. Cells were then incubated at $37^{\circ} \mathrm{C}, 5 \% \mathrm{CO}_{2}$ and fixed after 24 and $72 \mathrm{hr}$ in $10 \%$ formalin in order to assess attachment and morphology. Glass coverslips were used as control. After washing with PBS, cells were permeabilized with $0.1 \%$ Triton-X/0.1\% Tween in PBS (PBST) and incubated in $1 \% \mathrm{w} / \mathrm{v}$ bovine serum albumin (BSA) blocking solution for $30 \mathrm{~min}$ at room temperature. Monoclonal anti-vinculin antibody (Sigma; 1:400) was left to react for $1 \mathrm{hr}$ at room temperature. Next, the samples were washed again with PBS and incubated with the secondary antibody (AlexaFluor594 goat anti-mouse, LifeTechnologies; 1:1,000) for $1 \mathrm{hr}$ in the dark. In order to visualize the cytoskeleton, cells were stained with phalloidin (AlexaFluor488, LifeTechnologies; 1:40). Samples were prepared on glass slides using ProLong Diamond Antifade mountant with 4',6-diamidino-2-phenylindole (DAPI; LifeTechnologies) and imaged using a fluorescence microscope (Leica DMI3000B).

To quantify cells proliferation, five fields of view per sample were randomly acquired at $10 \times$ magnification by an examiner blinded to the study groups. Cell nuclei were counted with the ImageJ software using the "analyze particle" tool (Schneider, Rasband, \& Eliceiri, 2012).

\subsection{Fabrication of fibrin gels and cell encapsulation}

Fibrin gels were prepared by mixing a fibrinogen-based solution (16$\mathrm{mg} / \mathrm{mL}$ fibrinogen [Milan], 34- $\mu \mathrm{g} / \mathrm{mL}$ aprotinin [Roche], and 100,000 cells) and an enzymatic solution $(4-\mathrm{U} / \mathrm{mL}$ thrombin [Roche], $8-\mathrm{U} / \mathrm{mL}$ Factor XIIla [Behring], $10-\mathrm{mM} \mathrm{CaCl}_{2}$ [Sigma]) in equal proportions. A final volume of $100 \mu \mathrm{l}$ was dispensed in home-made PDMS wells (0.7 $\mathrm{mm}$ in diameter) and the final system was incubated for $1 \mathrm{hr}$ at $37{ }^{\circ} \mathrm{C}, 5 \% \mathrm{CO}_{2}$ to complete the fibrin gelation. The cell-hybrid gels were transferred into 48-well plates, covered with medium and incubated for further analyses.

To study the influence of laminin on the response of encapsulated ASC, different concentrations ranging between 1 and $100 \mu \mathrm{g} / \mathrm{mL}$ were added to the initial fibrinogen-based solution.

\subsection{Cell distribution and morphology in the matrix}

After $24 \mathrm{hr}$ of culture, cells were washed with sterile PBS and fixed in $10 \%$ formalin for $20 \mathrm{~min}$ at room temperature. The gels were then washed again and permeabilized in PBST for $30 \mathrm{~min}$ at room temperature. Following several additional rinses, non-specific antigens were blocked with $1 \%$ BSA solution for 30 min and cells were stained with phalloidin to visualize F-actin fibres. After 20 min of incubation in the 
dark, the gels were washed to remove any unreacted staining solution and stored in PBS at $4{ }^{\circ} \mathrm{C}$ for further analysis.

Images were acquired in PBS using confocal microscopy (Zeiss LSM 700) and z-stacks were finally reconstructed with the Zen Software (Zeiss, v. 2012, black edition) and ImageJ.

\subsection{Cell viability and proliferation}

Cell viability over time was studied by using a live/dead cell viability kit (LifeTechnologies). After cells' incubation for 1, 4, and 7 days, samples were rinsed in sterile PBS and a solution containing 2-mM ethidium homodimer-1 and 4-mM Calcein-AM was added to each gel. Samples were incubated for $30 \mathrm{~min}$ at room temperature in the dark, followed by rinsing with warm PBS to remove any unreacted dye. The gels were then observed with a fluorescence microscope and cell viability was expressed as the percentage of live cells over the total number of cells in each gel $(n=3$, with an average of five random fields of view per sample).

In addition, cell metabolic activity was monitored over 4 days using the AlamarBlue (AB) assay solution ( $n=3)$, according to the manufacturer protocol (AbD Serotec). Briefly, a $10 \%$ AB solution was added to each sample, including negative controls with medium only and incubated at $37{ }^{\circ} \mathrm{C}, 5 \% \mathrm{CO}_{2}$ for $4 \mathrm{hr}$ avoiding light exposure. Aliquots of the reacted solution were transferred in a new transparent 96-well plate, and the absorbance was read at 570 and $600 \mathrm{~nm}$ with a plate reader (Tecan Saphyre II). The cell metabolic activity was then expressed as the percentage reduction of the $A B$ reagent, calculated as

$$
\% A B \text { reduction }=\frac{\left(\varepsilon_{\text {ox_600 }} \times A_{570}\right)-\left(\varepsilon_{\text {ox_5570 }} \times A_{600}\right)}{\left(\varepsilon_{\text {red_570 }} \times C_{600}\right)-\left(\varepsilon_{\text {red_600 }} \times C_{570}\right)} \times 100
$$

with $A_{570}$ and $A_{600}$ being the absorbance values of each sample at 570 and $600 \mathrm{~nm}$, respectively, $C_{570}$ and $C_{600}$ being the absorbance values of the negative control; $\varepsilon_{\text {ox_600 }}=117,216, \varepsilon_{\text {ox_570 }}=80,586$, $\varepsilon_{\text {red_600 }}=14,652$, and $\varepsilon_{\text {red_570 }}=155,677$, the molar extinction coefficients of the $A B$ reagent in the oxidized and reduced form.

\subsection{Cells' interaction with laminin}

The expression of laminin receptors on ASC membranes as a function of laminin concentration in the gels was investigated through fluorescence staining. Following fixation in $10 \%$ formalin, the cells membrane was permeabilized and non-specific antigens were blocked with $1 \%$ BSA solution as previously described. Samples were then incubated for $1 \mathrm{hr}$ with a solution of rabbit anti-laminin and mouse anti-67KDa laminin receptor antibodies (Abcam; 1:200 and 1:50, respectively) to stain, respectively, laminin and its membrane receptor. Following PBS rinsing, the specific secondary antibodies (AlexaFluor488 donkey anti-rabbit and AlexFluor594 goat antimouse) were conjugated for $1 \mathrm{hr}$ in the dark before staining the nuclei with a $0.6-\mu \mathrm{g} / \mathrm{mL}$ DAPI solution (Sigma) for $5 \mathrm{~min}$. Samples were finally rinsed with PBS before imaging with confocal microscopy.

\subsection{In vitro coculture of DRG explants and stem cells}

The interaction of nerve tissue with fibrin gels was simulated in vitro with cultures of DRG explants. Gels were prepared as previously described (\$2.3) and divided in four different groups with/without laminin (1 $\left.\mu \mathrm{g} / \mathrm{mL} ; \mathrm{LN}^{+} \mathrm{LN}^{-}\right)$and in presence/absence of $\mathrm{ASC}\left(\mathrm{ASC}^{+} / \mathrm{ASC}^{-}\right)$.

After rat euthanasia, the spinal column was carefully removed and divided in half along the longitudinal axis. DRG were pulled out of the vertebral canals and maintained in $\mathrm{Ham}^{\prime} \mathrm{s}$ F12 medium (LifeTechnologies) supplemented with 1\% PS. DRG roots were cleaned under a dissection microscope and transferred on top of the gels, gently pushing to ensure tissue adhesion. Dulbecco's Modified Eagle Medium/Nutrient Mixture F-12 (DMEM/F-12, 1:1, GlutaMAX, LifeTechnologies) medium supplemented with $50 \mathrm{ng} / \mathrm{mL}$ of nerve growth factor (NGF 2.5S, Millipore) was added to each well containing a DRG explant, and the system was incubated at $37{ }^{\circ} \mathrm{C}, 5 \% \mathrm{CO}_{2}$. All explants were cultured for up to 6 days, changing the medium every 2 days, and fixed in $10 \%$ formalin solution for 40 min for further immunocytochemistry analysis. The explants were permeabilized and stained against mouse $\beta$-Tubulin (Abcam; 1:500) to visualize neurites. Following PBS rinsing, AlexaFluor488 goat anti-mouse was conjugated and the cell nuclei were stained with a $0.6 \mu \mathrm{g} / \mathrm{mL}$ DAPI. Tissues were finally imaged by confocal microscopy.

Fibrin gels were also prepared directly in PDMS conduits $(1.6 \mathrm{~mm}$ in diameter). In case of cell encapsulation, ASC were previously stained for $30 \mathrm{~min}$ in a serum-free growth medium with a CellTracker solution (5 $\mathrm{MM}$, Orange CMTMR, LifeTechnologies), then washed with sterile PBS and encapsulated in the gels on the day of the experiment. DRG explants were transferred on top of each preformed gel in the PDMS tube and cultured for up to 6 days to visualize satellite cells (rabbit anti-S100; AlexaFluor594 donkey anti-rabbit) and neurites, respectively. Tissues were finally imaged by confocal microscopy.

\section{8 | In vivo study}

\subsection{1 | Conduit implantation}

Peripheral nerve regeneration was studied using the rat sciatic nerve model, in accordance with the local veterinary commission of the canton of Vaud. Male Sprague-Dawley rats (Janvier, France) weighing $250 \pm 30 \mathrm{~g}$ were selected. Animals were divided in three different groups, all including PDMS bridging conduits filled with fibrin gels and an additional condition described hereafter: ASC only, laminin $(1 \mu \mathrm{g} / \mathrm{mL})$ only, and ASC + laminin, abbreviated as GC, L, and LC ( $n=5)$.

All gels were prepared on the day of implantation and loaded directly in the final PDMS conduits (length $=17 \mathrm{~mm}$; inner diameter $=2 \mathrm{~mm}$ ) as previously described (§2.7) and incubated at $37{ }^{\circ} \mathrm{C}$, $5 \% \mathrm{CO}_{2}$ in a-MEM medium until surgery. In case of cell transplantation, $1 \times 10^{6}$ cells were seeded in each conduit.

All surgical implantations were performed on the left sciatic nerve in aseptic conditions using a power focus surgical microscope (Leica M80). After having induced deep anaesthesia with isofluorane (Attane $^{\mathrm{TM}}$, Piramal Enterprises Ltd.), a skin incision from the left knee to the hip was made for exposure of the underlying muscles, which were then retracted to reveal the sciatic nerve (di Summa et al., 
2013). The sciatic nerve was transected, and nerve ends were fixed to the conduit by two epineural sutures on each slide (9/0 Prolene, Ethicon); proximal and distal nerve stumps were inserted $1 \mathrm{~mm}$ into the tube thus leaving a 15-mm gap. Muscles and fascia layers were closed with single resorbable stitches (3/0 Monocryl, Ethicon), and the skin by a continuous subcuticular running suture. All experimental groups were housed on sawdust, with a 12-hr light:12-hr dark cycle and received food and water ad libitum.

\subsection{2 | Tissue harvesting and analysis}

Animals were perfused with $4 \%$ paraformaldehyde after 8 weeks. Tissues were further fixed overnight and washed in PBS, gently removed from the PDMS conduits and embedded in paraffin. Cross sections of the regenerated nerve ( $4 \mu \mathrm{m}$ thick) were obtained by cutting three to four slices every millimetre along both proximal and distal nerve stumps and collected on glass slides (Superfrost plus, Menzel-Gläser) and stored at $4{ }^{\circ} \mathrm{C}$. Following dewaxing and antigen retrieval in citrate buffer $(\mathrm{pH}=6)$, tissues were washed in PBS and blocked in 1\% BSA solution for $1 \mathrm{hr}$ at room temperature. The primary antibodies (rabbit anti-S100 [Sigma]; 1:200) and mouse anti-Neurofilament 200 kDa (Millipore; 1:200) were then incubated overnight at $4{ }^{\circ} \mathrm{C}$. Secondary antibodies (AlexaFluor594 donkey anti-rabbit and AlexaFluor488 goat anti-mouse) were conjugated the day after for $1 \mathrm{hr}$ at room temperature. Coverslips were finally applied with a DAPI mounting medium and samples were imaged with a fluorescence microscope.

Using the set of nerve cross sections obtained approximately $1 \mathrm{~mm}$ from the stitches of the distal nerve stump, regenerated axons were identified and quantified using ImageJ (5 random fields selected per animal), evaluating final number of fibres per square millimetres, single fibre area and percentage of regenerated fibres.

\section{9 | Statistical analysis}

Results are expressed as mean \pm standard error of the mean. Statistical significance was estimated by one- and two-way analysis of variance tests in Prism 5 (GraphPad Software v.5.03). Bonferroni's post-test was performed to compare groups. Levels of significance were expressed as $p$ values $\left({ }^{*} p<.05,{ }^{* *} p<.01,{ }^{* * *} p<.001\right)$. Additionally, $t$ tests were performed to compare proximal and distal stump results obtained from the in vivo study per each group. Statistical significance $(p<.05)$ was indicated with \#.

\section{3 | RESULTS}

\subsection{ASC proliferation on PDMS substrates as function of adhesive proteins}

During the phase of cell attachment (first $24 \mathrm{hr}$ of culture), only $15 \%$ of cells were observed on untreated PDMS substrates compared with glass coverslips $\left(\sim 50 \% ;{ }^{* * *} p<.001\right)$. Cells presented a very round and contracted morphology, with limited spreading (Figure 1b). In contrast, ASC on our control surface presented good spreading, cytoskeleton development and formation of focal adhesions (Figure 1e).

ASC response on fibronectin- and laminin-coated PDMS surfaces resulted in much higher attachment $(>90 \%$ on both proteins; ${ }^{* * *} p<.001$ ), also confirmed by fluorescence images (Figure 1c,d). Following 3 days of culture, laminin-coated discs displayed higher proliferation compared with fibronectin-coated discs $\left({ }^{* * *} p<.001\right)$, almost doubling the initial cell number (Figure 1a). The beneficial effect of adhesive proteins, in particular laminin, was therefore confirmed, reaching cell number 10 -fold higher than untreated PDMS substrates.

\section{2 | Cell encapsulation in 3-D fibrin matrices}

ASC in fibrin gels presented 3-D-stellar morphology and well-developed cytoskeletal structures after $24 \mathrm{hr}$ of culture (Figure 2a). The addition of laminin confirmed our findings from the 2-D in vitro culture: Spreading and projection of cell extensions improved, also probably due to the increase in cell number (Figure 2b). Overall, encapsulated cells were uniformly distributed throughout the volume of the scaffold (Figure 2c,d).
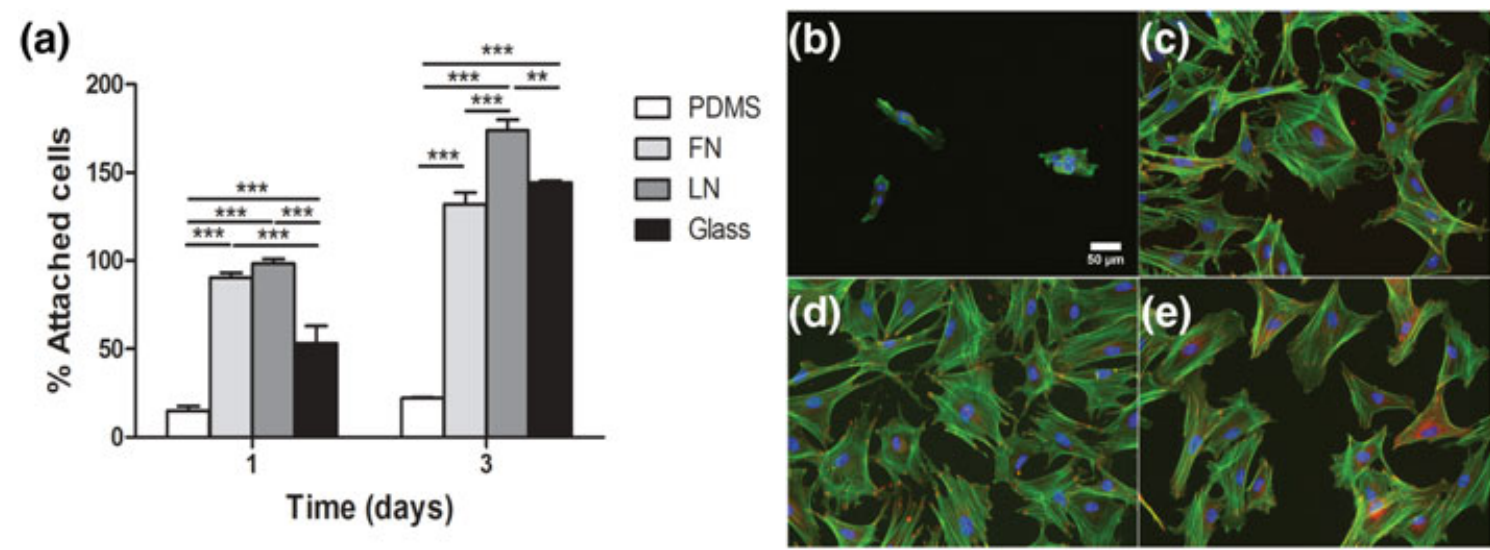

FIGURE 1 In vitro adipose-derived stem cells response as function of adhesive proteins. (a) Cell attachment and proliferation on different substrates over 3 days of culture. Levels of significance were expressed as $p$ values $\left({ }^{* *} p<.01,{ }^{* * *} p<.001\right)$. Cell morphology after 24 hr was studied by immunocytochemistry, staining the cells for vinculin (red, AlexaFluor594), phalloidin (green, AlexaFluor488), and nuclei (blue, 4',6diamidino-2-phenylindole). Fluorescence images of adiposed-derived stem cells seeded on (b) polydimethylsiloxane (PDMS), (c) fibronectin (FN)coated PDMS, (d) laminin (LN)-coated PDMS, and (e) glass coverslips. Scale bar $=50 \mu \mathrm{m}$ 


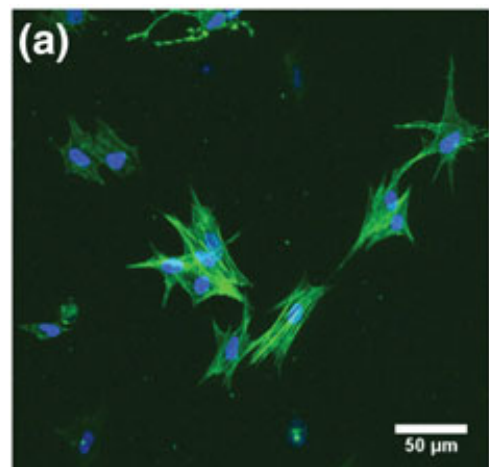

(e)
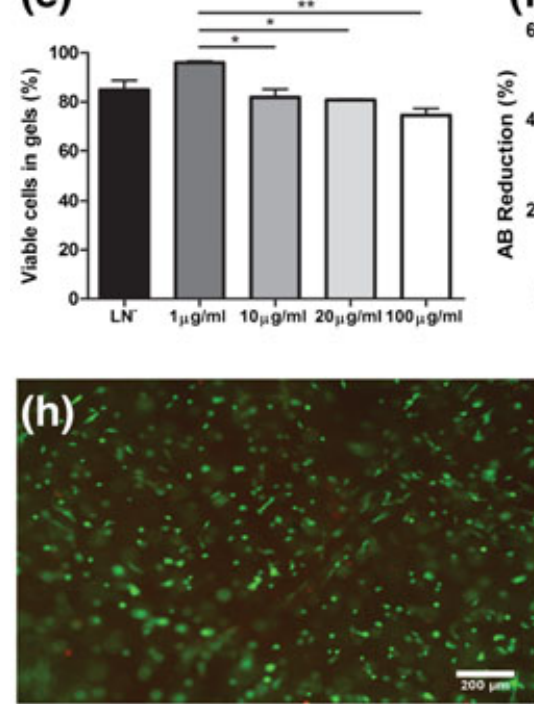

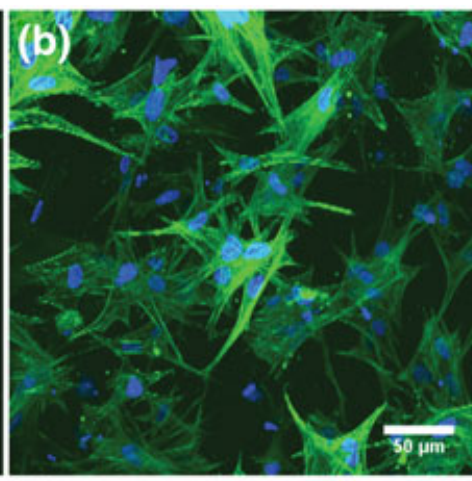

(f)

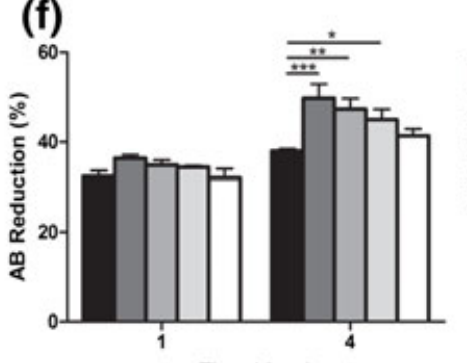

Time (days)

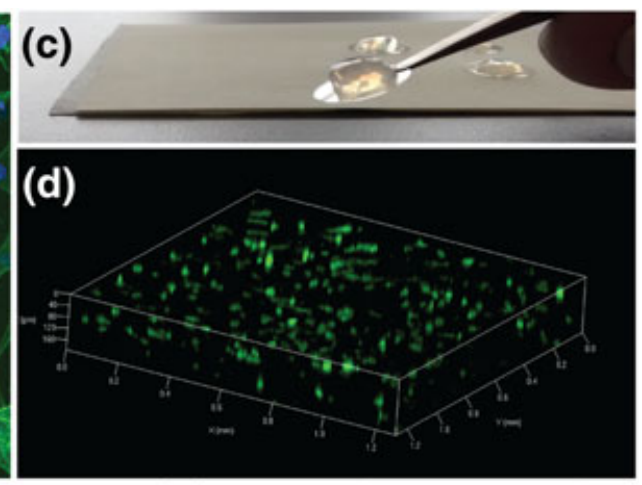

(g)

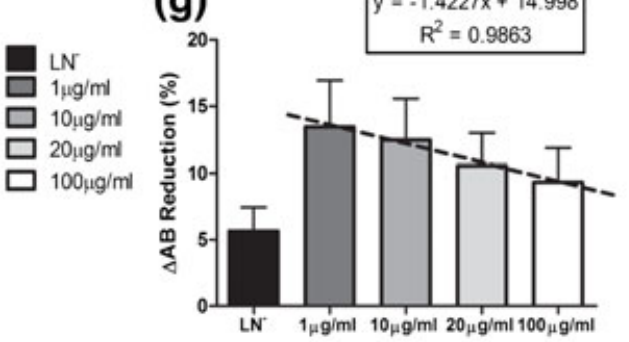

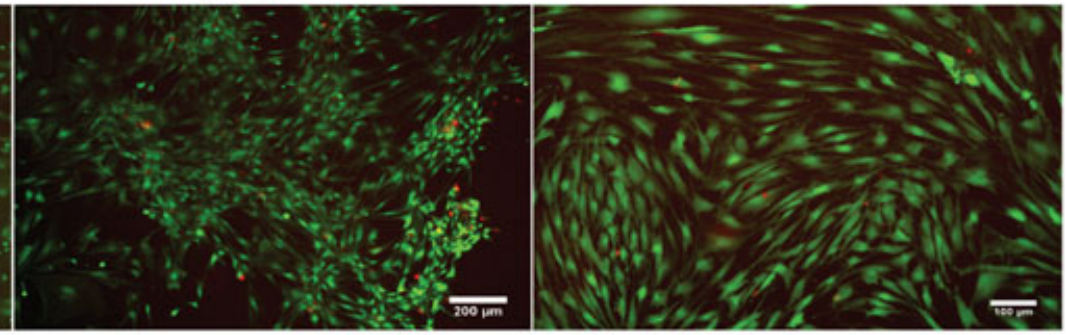

Days 4

FIGURE 2 In vitro ASC encapsulation and viability in fibrin gels, from laminin-free ( $\mathrm{LN}^{-}$) to laminin ranging between 1 and $100 \mu \mathrm{g} / \mathrm{mL}$. Encapsulated cells in (a) absence or (b) presence of laminin, respectively, stained for phalloidin (green, AlexaFluor488) and nuclei (blue, 4',6diamidino-2-phenylindole), scale bar $=50 \mu \mathrm{m}$. (c) Gels presented a well-defined shape after gelation in home-made polydimethylsiloxane molds. (d) Three-dimensional confocal reconstruction of a defined volume of the cell-fibrin system (section thickness $=200 \mu \mathrm{m}$ ). (e) Cell viability was measured with the live/dead assay. (f) Cell proliferation was expressed as percentage of AlamarBlue (AB) reduction over 4 days of culture. (g) The proliferation rate $(\triangle \mathrm{AB})$ was then approximated to a linear equation. Levels of significance are expressed as $p$ values $\left({ }^{*} p<.05,{ }^{* *} p<.01,{ }^{* * *} p<.001\right)$. (h) Evolution of encapsulated cells over 7 days, migrating from the gels towards the bottom of the wells

\section{3 | Cell viability and proliferation in fibrin gels}

Fibrin gels did not affect cell viability in the first $24 \mathrm{hr}$ of culture (Figure $2 \mathrm{e}$ ), with more than $80 \%$ of cells surviving after encapsulation. No differences were observed between laminin-free gels and those supplemented with $1-\mu \mathrm{g} / \mathrm{mL}$ laminin. However, a gradual reduction in cell viability was recorded at higher protein concentrations (>10 $\mu \mathrm{g} / \mathrm{mL}$ ), suggesting a potential toxicological effect of elevated laminin levels in the system. Viable cells retained their proliferative activity over time and started to migrate towards the bottom of the wells in which the hybrid systems were cultured. Following 7 days of culture, most of the gel was degraded. Migrated cells were fully viable in the confined environment (Figure $2 \mathrm{~h}$ ).

We monitored cell proliferation at different time points through the recording of cell metabolic activity, expressed as a percentage of the reagent reduction (Figure 2f). No difference between groups was measured after the first $24 \mathrm{hr}$ of culture $(p>.05)$. However, the presence of laminin in the gels favoured cell proliferation at later time points: The lower the protein concentration, the higher the proliferation (Figure $2 \mathrm{~g}$ ). Low concentrations of laminin, that is, $1 \mu \mathrm{g} / \mathrm{mL}$, resulted in the highest proliferation rates compared with the other groups $\left({ }^{* * *} p<.001\right)$.

Direct correspondence between the laminin concentration $(\mathrm{x})$ and the proliferation rate $(\triangle A B)$ was observed and approximated to a linear profile such as $c=a+b x$, in which $c$ is the proliferation rate, $a$ is the coefficient of cell proliferation in fibrin gels, and $b$ is a coefficient related to the specific gel properties and culture conditions. We calculated $a=15.00$ and $b=-1.42$, with a good approximation to a regression line $\left(r^{2}=.97\right)$.

\section{4 | Cell-protein interaction}

The expression of the $67 \mathrm{kDa}$ laminin receptor (67LR) and the interaction between ASC and laminin in the gels was investigated through immunocytochemistry. The 67LR receptor 
was detected at low concentrations in all the gels with laminin, indicating that these receptors are important for cell-protein interactions (Figure 3).

Laminin distribution in the cell-fibrin system was instead unexpected. Fibrin gels at low laminin concentrations $(1 \mu \mathrm{g} / \mathrm{mL})$ presented higher levels of the same protein compared with more highly supplemented gels. It could be therefore hypothesized that lower laminin concentrations stimulated further the synthesis of the same protein by the cells while encapsulated and cultured in the gels up to 4 days. This would in turn explain the increasing expression of the 67LR receptor.

\section{5 | DRG explants and interaction with fibrin gels}

DRG explants strongly adhered on laminin-coated glass coverslips and were cultured up to 6 days (Figure 4a). After only few days, it was
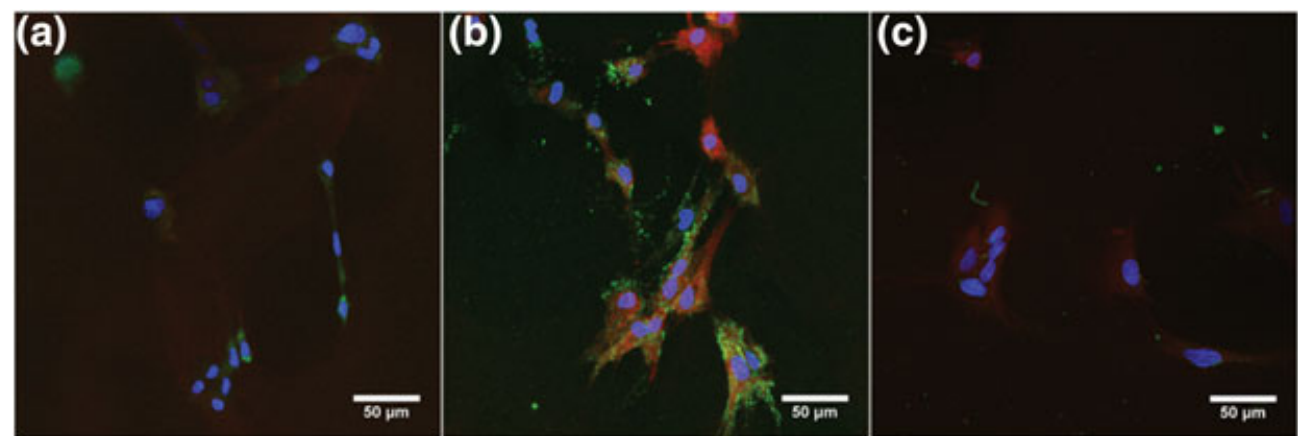

FIGURE 3 In vitro adipose-derived-stem-cells-laminin interactions observed by confocal microscopy. Cells were stained after 4 days of culture for the 67LR laminin receptor (red, AlexaFluor594), laminin (green, AlexaFluor488) and nuclei (blue, 4',6-diamidino-2-phenylindole). Fibrin gels were prepared with (a) $0-\mu \mathrm{g} / \mathrm{mL}$ laminin, (b) $1-\mu \mathrm{g} / \mathrm{mL}$ laminin, and (c) $20-\mu \mathrm{g} / \mathrm{mL}$ laminin. Scale bar $=50 \mu \mathrm{m}$
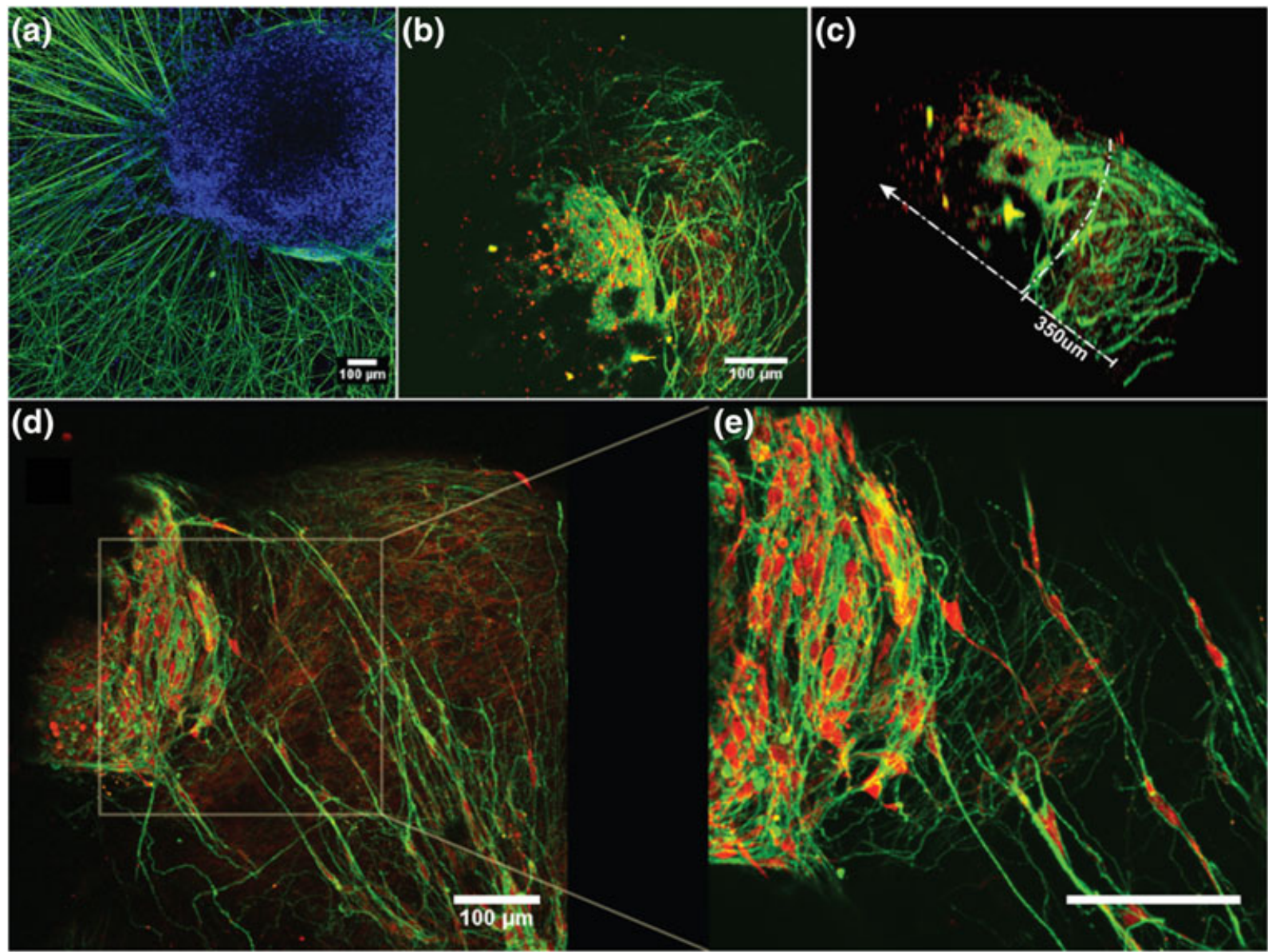

FIGURE 4 Confocal imaging of dorsal root ganglia explants on a surface and in 3-D scaffolds. (a) Culture of a dorsal root ganglia explant on laminincoated glass coverslips (staining: $\beta$-tubulin [green, AlexaFluor488] and nuclei [blue, 4',6-diamidino-2-phenylindole]). (b) Top view of dorsal root ganglia explant in a fibrin gel and (c) its rotated 3-D projection. (d,e) Zoomed images of dorsal root ganglia explant and glial cells' migration in a fibrin gel (staining: $\beta$-tubulin [green, AlexaFluor488] and S100 [red, AlexaFluor594]). Scale bar $=100 \mu \mathrm{m}$ 
possible to observe neurites projection extending from the DRG body. After 6 days, DRGs formed a very dense mat of neurites all around the main body, stabilizing the explant adhesion onto the substrate. However, satellite cells were mainly localized around the body, with limited migration through the neurites mat.

DRG explants were then cultured in a 3-D system, having the fibrin matrix confined to a PDMS tube with a similar size than a nerve guidance conduit. Three-dimensional gels did not obstruct neurite sprouting as shown in Figure $4 \mathrm{~b}-\mathrm{e}$. Interestingly, the elongation of neurites occurred in two directions: First, the neurites tended to reach the tube borders, and then they grew along the walls. It was observed that fibrin gels also supported cell migration from the DRG explant, with immunofluorescence showing colocalization of satellite cells (in red) on neurite projections, directed through the fibrin gel (Figure 4d,e).

\subsection{Coculture of DRG explants and fibrin- encapsulated ASC}

The presence of pre-encapsulated ASC in the fibrin gels seemed to improve neurite sprouting from DRG explants after 6 days of culture. Adding laminin in the gel strengthened further neurite outgrowth. (Figure 5a). Unfortunately, due to imaging limitations, no quantification was possible, but only a representative view of neurites growth is presented here.

When explants were cultured in tubes (Figure $5 b$ ), as previously observed after the live/dead assay, encapsulated ASC migrated out of the gel during the process of matrix degradation to finally grow on the supporting substrate. Similarly, in this DRG + fibrin-encapsulated ASC system, cells migrated toward the silicone tube wall, driving neurites through the longitudinal tube axis (Figure 5c).

\section{7 | In vivo regeneration of the sciatic nerve}

All animals survived the surgical procedure and recovered from anaesthesia. No autotomy complications occurred. Following tissue harvest after 8 weeks of implantation, no fibrin debris was observed in any conduit. Proximal and distal nerve stumps could be easily identified and removed from the external tubes.

Nerve regeneration was evaluated through analysis of immunohistological images (Figure 6a) and expressed as a measure of nerve fibres' density (fibres per millimetres and fibre area percentage; Figure 6b,c).

Upon analysis of the distal stumps across all groups, we observed the fewer and thinner nerve fibres in the GC group, where the fibres' density was significantly reduced $\left({ }^{\#} p<.05\right)$ compared with the other two groups; this suggests the presence of ASC alone is not sufficient to support robust nerve regeneration.

In contrast, we observed a higher density of nerve fibres at the distal stump when ASC were combined with laminin. ( $p<.05)$. This combination of biochemical and biological cues supported significant regenerative outcomes compared with the single cues. Proximal fibres were more robust $\left({ }^{\#} p<.05\right.$; Figure $\left.6 \mathrm{~d}\right)$ ), and fibres ${ }^{\prime}$ density at both stumps was comparable.

\section{4 | DISCUSSION}

ECM proteins like fibronectin and laminin are the main components of the basal lamina that surrounds the axons and nerve fascicles. They are also actively involved in tissue development during the embryonic phase. These molecules are defined as adhesion proteins because of their characteristic amino acid domains that particularly favour cell adhesion and spreading, such as Arg-Gly-Asp and Ile-Lys-Val-Ala-Val, respectively. Their use in surface coatings or bio-functionalization for

(a)
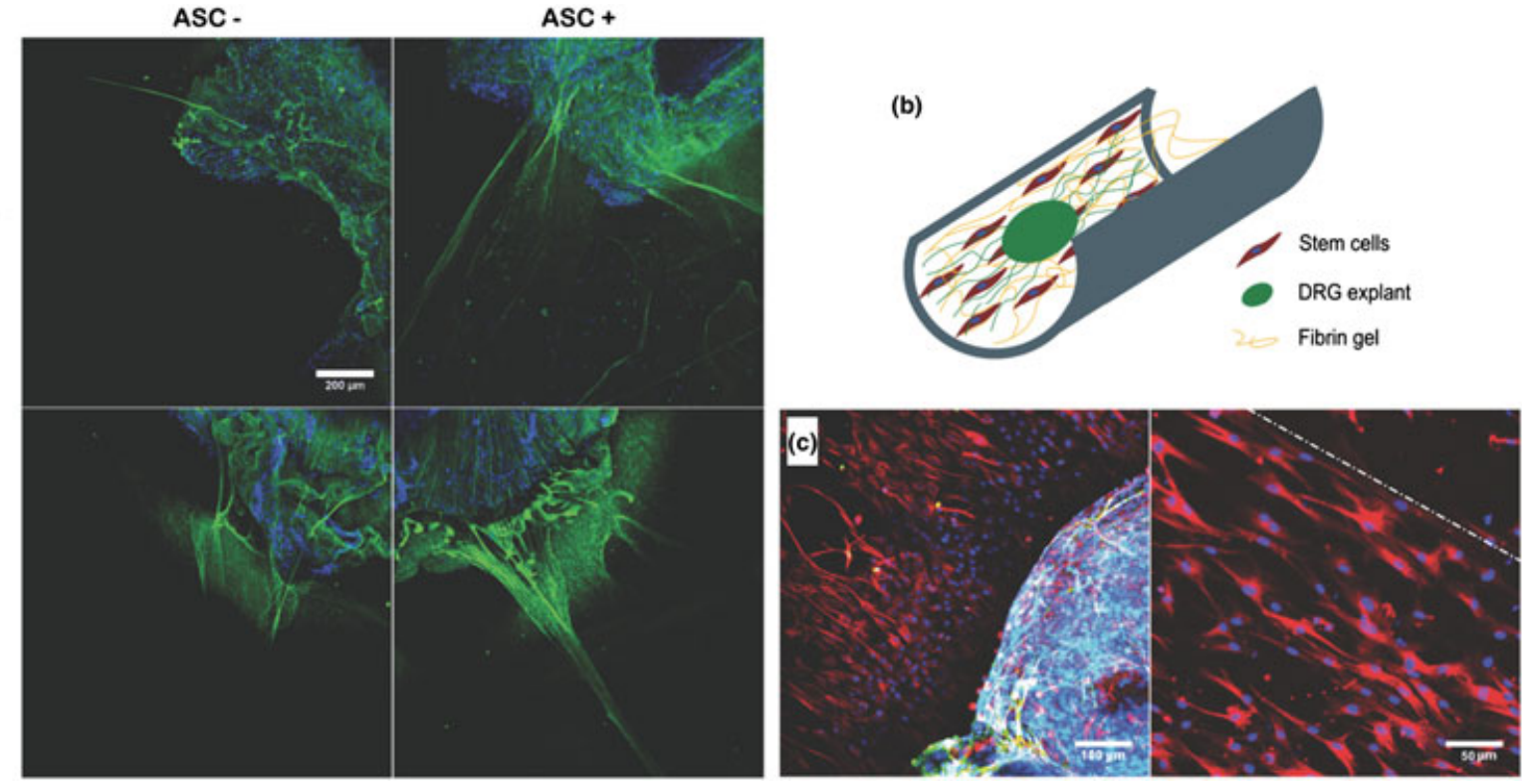

FIGURE 5 In vitro dorsal-root-ganglia-adipose-derived-stem-cells (DRG-ASC) interactions in the fibrin gel. (a) Fluorescence images of DRG explants grown in gels loaded with different conditions including ASC, laminin (LN), and a combination of the two factors. All four images were taken with the same magnification. (b) Schematic of the DRG-ASC coculture in polydimethylsiloxane conduits. (c) Top view of ASC growing within the polydimethylsiloxane conduit and in contact with the DRG explant (the white line indicates the direction of the tube longitudinal axis) 


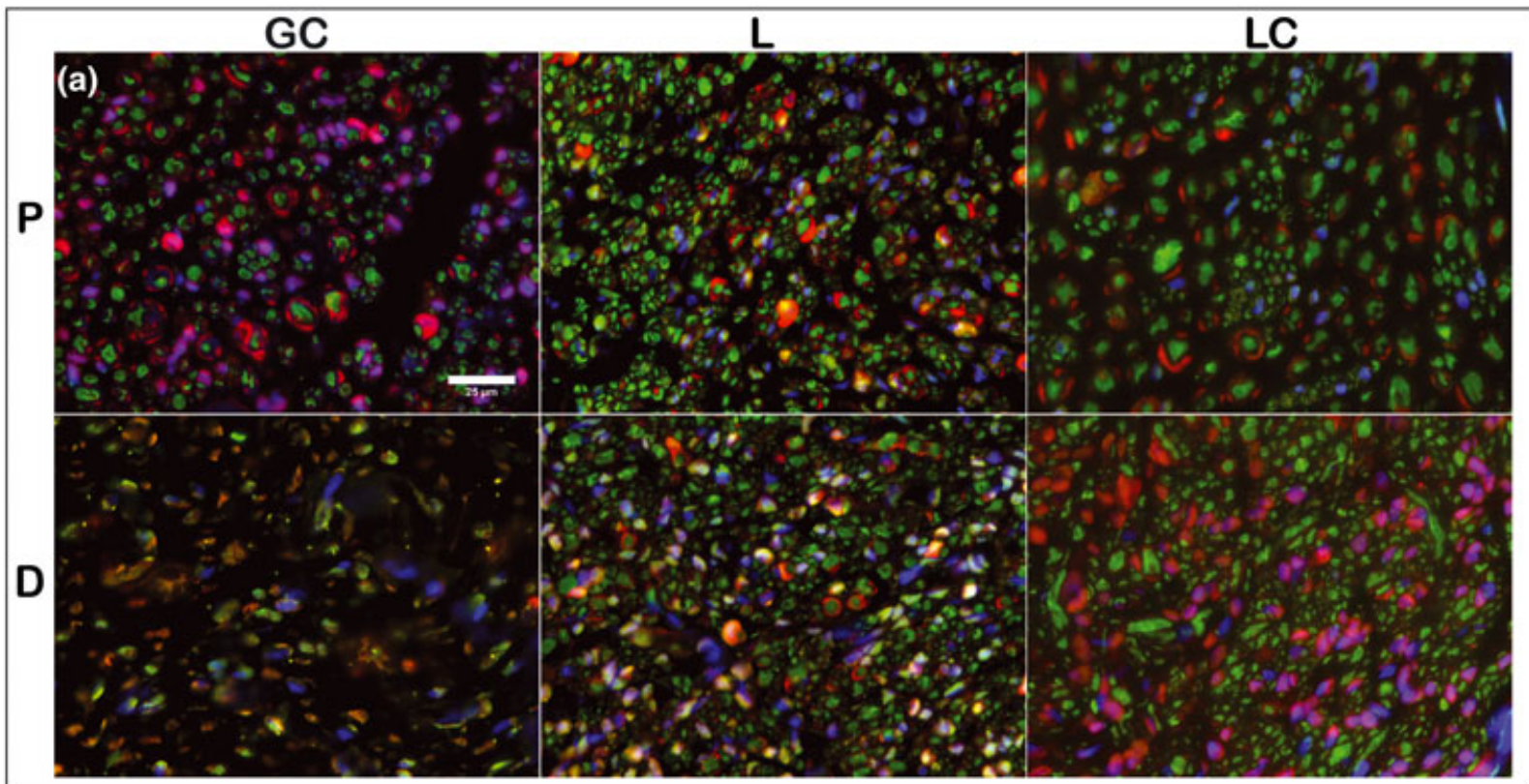

(b)

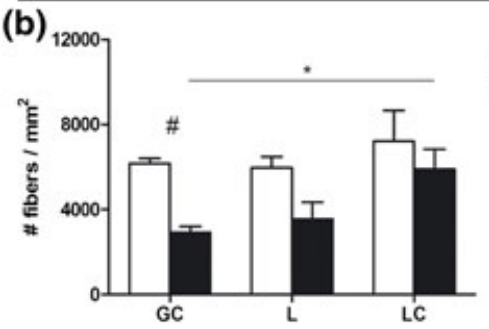

(c)

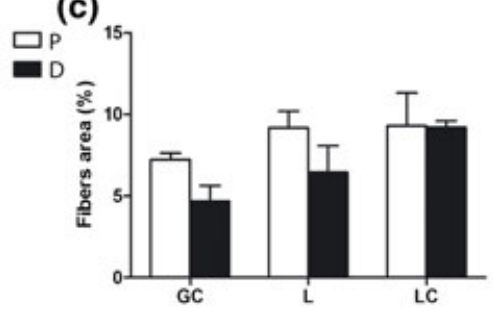

(d)

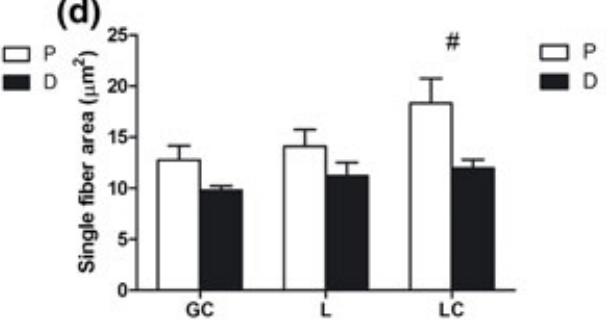

FIGURE 6 Sciatic nerve regeneration following complete transection. (a) Representative semithin sections of proximal (P) and distal (D) stumps of regenerated sciatic nerves. The sections were stained against S-100 (red), Neurofilament (green), and 4',6-diamidino-2-phenylindole (blue). The conduits embedded gels with adipose-derived stem cells only (GC), with laminin (L) only, and a combination of the two factors (LC). Scale bar $=25 \mu \mathrm{m}$. Nerve regeneration was quantified as (b) the number of fibres per square millimetres, (c) percentage of regenerated fibres, and (d) surface area of a single fibre. Values compared between proximal and distal stumps were considered statistically different when ${ }^{\#} p<.05,{ }^{*} p<.05$

applications in nerve repair has been deeply investigated in vitro in order to improve cell-material interaction and design a favourable scaffold for tissue regeneration (Rao \& Winter, 2009). Poly- $\varepsilon$-caprolactone films were functionalized with Arg-Gly-Asp-based sequences resulting in higher SC attachment and proliferation, as well as longer neurite sprouting from dissociated DRG neurons (de Luca, Faroni et al., 2016; de Luca, Stevens et al., 2013). Similar findings were obtained using laminin-derived peptides, which targeted specific neural responses system (Hosseinkhani et al., 2013; Tashiro et al., 1989). The importance of ECM molecules on glial and neuronal cells was also investigated using the complete proteins' sequence, confirming beneficial effects on cell survival, particularly when cultured under stress conditions (di Summa et al., 2013). Since most of the studies in literature were conducted on standard culture plates, we investigated the influence of laminin and fibronectin coatings on PDMS substrates. Silicones are often used in nerve interfaces and are core materials for neuroprosthetic devices (Musick et al., 2015; Srinivasan et al., 2016), albeit biodegradable polymers are favoured in nerve regeneration strategies.

In vitro tests were conducted with ASC as a promising substitute to SC. Their beneficial influence to nerve repair was observed both in vitro and in vivo, resulting in neuronal protection and supported nerve regeneration (Reid et al., 2011). These effects could be most likely ascribed to their ability to produce and release growth factors such as nerve growth factor, vascular endothelial growth factor, and brain-derived neurotrophic factor (Luo et al., 2012; Sowa, Imura, Numajiri, Nishino, \& Fushiki, 2012; Zhao et al., 2009) in the confined environment of the nerve guidance tube. Each of these molecules is essential for the recruitment of endogenous $\mathrm{SC}$ at the site of a peripheral nerve injury, improving tissue regeneration and functional repair.

When ASC were seeded either on fibronectin- or laminin-coated substrates, their initial attachment over the first $24 \mathrm{hr}$ was four-fold enhanced compared with the bare PDMS. ECM molecules supported cell growth over time, confirming the importance of biochemical cues for cell viability. Compared with cells growing on standard tissue culture plastic, cell proliferation was not affected by the presence of these proteins (di Summa et al., 2013), while ECM molecules acted as key mediators for cell-material interactions in the case of the very hydrophobic and inert surface of PDMS. Between the two evaluated proteins, laminin promoted higher proliferation therefore was selected as the biochemical cue for the subsequent studies.

We next incorporated the laminin in a three-dimensional matrix. Among the few existing reports on the topic, Labrador et al. investigated the effect of laminin on nerve regeneration using the protein concentration contained in the matrigel composition and varying its concentration by dilution of the matrigel. They observed that nerve regeneration was improved by using highly diluted gels, which 
contained the lowest concentration of laminin; quantitative concentration values were not available (Labrador, Buti, \& Navarro, 1998). A few years later, Pittier et al. tested the effect of laminin-1 adsorption in fibrin gels on PC12 neurite extension. Concentrations as low as $20 \mu \mathrm{g} / \mathrm{mL}$ were successful in promoting long neurite outgrowth and improved cell response (Pittier, Sauthier, Hubbell, \& Hall, 2005). Higher laminin concentrations $(60 \mu \mathrm{g} / \mathrm{mL})$ loaded in agarose gels were also found important in strategies to bridge nerve injured gaps ( $\mathrm{Yu} \&$ Bellamkonda, 2003). Here, we have demonstrated that the lower the protein concentration, the better the viability and survival of adipose stem cells in fibrin gels. Laminin concentrations ranging between 1 and $100 \mu \mathrm{g} / \mathrm{mL}$ were tested, and concentrations as low as $1 \mu \mathrm{g} / \mathrm{mL}$ resulted in the highest ASC viability and proliferation compared with higher contents of the ECM protein or plain fibrin gels. Further investigations are now needed to clarify the apparent "toxic" effect of the protein in high concentration (>10 $\mu \mathrm{g} / \mathrm{mL}$ ) on ASC and surrounding environment.

Axon regeneration through fibrin gels was simulated in vitro using DRG explants. We observed the degradation of the gels activated by the migration and proliferation of satellite cells, as well as of ASC when cocultured in the hybrid system. As reported by Man et al., proliferating cells secrete high levels of serine proteases and matrix metalloproteinases, inducing fibrinolysis (Man, Davis, Itoh, Leach, \& Bannerman, 2011). In turn, the outgrowth of neurites from DRG explants is directly dependent upon the degradation rate of the gels. Even if cellular dynamics may be difficult to evaluate using immunofluorescence images, the regeneration model may be explained as a cascade of events: First fibrinolysis is activated by the pre-encapsulated ASC and surrounding cells migrating from the nervous tissue; next, the degrading fibrin matrix is substituted by the endogenous production of a newly formed fibrin membrane; finally, the transplanted ASC assist the native $\mathrm{SC}$ during the regenerative pathway of the damaged tissue. When laminin is further supplied to the system, the growth of thicker and denser neurites is observed, suggesting potential robust nerve regeneration in vivo. We further confirmed our in vitro results using a rat sciatic nerve model over 8 weeks implantation.

ASCs embedded in a fibrin matrix were previously shown to significantly enhance regeneration of peripheral nerve injuries after primary coaptation in rats (Reichenberger et al., 2016). Here, we tested the effect of the hybrid cell-gel cue against the regeneration of peripheral nerves across long (15 mm long) gaps, a clinically relevant challenge. Biological cues in the lumen of the conduits are necessary to promote advanced regeneration in long gaps. Wang et al. demonstrated that the inclusion of basic fibroblast growth factor in biodegradable tubes reduced the delay of axonal regeneration observed across a $15-\mathrm{mm}$ gap in the control group without growth factors (Wang et al., 2003). Engineered matrices containing ECM components and transplanted SC and SC-like differentiated ASC resulted in three-fold greater regeneration than empty conduits (Georgiou, Golding, Loughlin, Kingham, \& Phillips, 2015; Georgiou et al., 2013). In this study, we observed that undifferentiated ASC alone were not able to support high extents of regeneration, probably because the long injury gap hindered the supply of nutrients in the lumen of the nonpermeable PDMS conduit. However, in the presence of ECM proteins within the gel, we observed a significant increase of fibres' sprouting at the proximal stump and significantly denser nerve fibres at the distal stump compared with the GC group. Despite potential bias from quantitative morphometric analysis, results seem to confirm the cumulative positive effect of fibrin gel-encapsulated ASC and laminin in this in vivo study poses promising perspectives for using ASC in peripheral nerve repair. Further investigations on the viability of transplanted cells and their interplay with ECM proteins are necessary to better understand the mechanisms of regeneration and develop a cell-based construct for nerve repair.

\section{5 | CONCLUSIONS}

Modification of 3-D matrices with ECM molecules is crucial for cell encapsulation. The presence of laminin contributed to ASC viability and proliferation following release in the confining environment due to fibrin degradation. However, this beneficial effect is inversely proportional to the protein concentration, and cell-laminin interactions are saturated at very low concentrations. Such cell-hybrid gel systems also supported neurite sprouting from DRG explants. In vivo tests confirmed the importance of combining ASC with ECM molecules in order to reinforce nerve regeneration. From these results, we could envisage that gradients of fibrin concentrations will induce cell release over time, guaranteeing a constant supply of biochemical cues during the regeneration process. Laminin-enhanced ASC-carrying gels are a potential path to improve the efficacy of PDMS guidance conduits and peripheral nerve electrode interfaces.

\section{ACKNOWLEDGEMENTS}

The authors would like to acknowledge the Swiss National Science Foundation for funding this research project (grant CR32I2_149609). The authors are also very grateful to $G$. Courtine and his research group, and Jessica Sordet-Dessimoz from EPFL Histology Core Facility for their valuable assistance during the in vivo study. The authors confirm that there are no conflicts of interest associated with this publication.

\section{ORCID}

S.P. Lacour $\odot$ http://orcid.org/0000-0001-9075-4022

\section{REFERENCES}

Carlson, K. B., Singh, P., Feaster, M. M., Ramnarain, A., Pavlides, C., Chen, Z. L., ... Strickland, S. (2011). Mesenchymal stem cells facilitate axon sorting, myelination, and functional recovery in paralyzed mice deficient in Schwann cell-derived laminin. Glia, 59, 267-277.

Chernousov, M. A., \& Carey, D. J. (2000). Schwann cell extracellular matrix molecules and their receptors. Histology and Histopathology, 15, 593-601.

Faroni, A., Smith, R. J. P., \& Reid, A. J. (2014). Adipose derived stem cells and nerve regeneration. Neural Regeneration Research, 9, 1341-1346.

Gattazzo, F., Urciuolo, A., \& Bonaldo, P. (2014). Extracellular matrix: A dynamic microenvironment for stem cell niche. Biochimica et Biophysica Acta, 1840, 2506-2519.

Georgiou, M., Bunting, S. C., Davies, H. A., Loughlin, A. J., Golding, J. P., \& Phillips, J. B. (2013). Engineered neural tissue for peripheral nerve repair. Biomaterials, 34, 7335-7343. 
Georgiou, M., Golding, J. P., Loughlin, A. J., Kingham, P. J., \& Phillips, J. B. (2015). Engineered neural tissue with aligned, differentiated adiposederived stem cells promoted peripheral nerve regeneration across a critical sized defect in rat sciatic nerve. Biomaterials, 37, 242-251.

Gomillion, C. T., \& Burg, K. J. L. (2006). Stem cells and adipose tissue engineering. Biomaterials, 27, 6052-6063.

Gordon, T., Tyreman, N., \& Raji, M. A. (2011). The basis for diminished functional recovery after delayed peripheral nerve repair. The Journal of Neuroscience, 31, 5325-5334.

Gu, X. S., Ding, F., \& Williams, D. F. (2014). Neural tissue engineering options for peripheral nerve regeneration. Biomaterials, 35(24), 6143-6156.

Hoke, A. (2006). Mechanisms of disease: What factors limit the success of peripheral nerve regeneration in humans? Nature Clinical Practice Neurology, 2, 448-454.

Hosseinkhani, H., Hiraoka, Y., Li, C. H., Chen, Y. R., Yu, D. S., Hong, P. D., \& Ou, K. L. (2013). Engineering three-dimensional collagen-IKVAV matrix to mimic neural microenvironment. ACS Chemical Neuroscience, 4, 1229-1235.

Ide, C. (1996). Peripheral nerve regeneration. Neuroscience Research, 25 , 101-121.

Kingham, P. J., Kalbermatten, D. F., Mahay, D., Armstrong, S. J., Wiberg, M. \& Terenghi, G. (2007). Adipose-derived stem cells differentiate into a Schwann cell phenotype and promote neurite outgrowth in vitro. Experimental Neurology, 207, 267-274

Kingham, P. J., Mantovani, C., \& Terenghi, G. (2011). Stem cell and neuron co-cultures for the study of nerve regeneration. Methods in Molecular Biology, 695, 115-127.

Labrador, R. O., Buti, M., \& Navarro, X. (1998). Influence of collagen and laminin gels concentration on nerve regeneration after resection and tube repair. Experimental Neurology, 149, 243-252.

Liu, G. B., Cheng, Y. X., Feng, Y. K., Pang, G. J., Li, Q., Wang, Y., ... Tong, X. J. (2011). Adipose-derived stem cells promote peripheral nerve repair. Archives of Medical Science, 7, 592-596.

Longo, M. V. L., Marques de Faria, J. C., Isaac, C., Nepomuceno, A. C. Teixeira, N. H., \& Gemperli, R. (2015). Comparisons of the results of peripheral nerve defect repair with fibrin conduit and autologous nerve graft: An experimental study in rats. Microsurgery. [Epub ahead of print]

de Luca, A. C., Faroni, A., Downes, S., \& Terenghi, G. (2016). Differentiated adipose-derived stem cells act synergistically with RGD-modified surfaces to improve neurite outgrowth in a co-culture model. Journal of Tissue Engineering and Regenerative Medicine, 10, 647-655.

de Luca, A. C., Faroni, A., \& Reid, A. J. (2015). Dorsal root ganglia neurons and differentiated adipose-derived stem cells: $\mathrm{An}$ in vitro co-culture model to study peripheral nerve regeneration. Journal of Visualized Experiments, 6, e52543.

de Luca, A. C., Stevens, J. S., Schroeder, S. L. M., Guilbaud, J. B., Saiani, A., Downes, S., \& Terenghi, G. (2013). Immobilization of cell-binding peptides on poly-e-caprolactone film surface to biomimic the peripheral nervous system. Journal of Biomedical Materials Research. Part A, 101 491-501.

Luo, H. L., Zhang, Y. J., Zhang, Z. Q., \& Jin, Y. (2012). The protection of MSCs from apoptosis in nerve regeneration by TGF beta 1 through reducing inflammation and promoting VEGF-dependent angiogenesis. Biomaterials, 33, 4277-4287.

Man, A. J., Davis, H. E., Itoh, A., Leach, J. K., \& Bannerman, P. (2011). Neurite outgrowth in fibrin gels is regulated by substrate stiffness. Tissue Engineering Part A, 17, 2931-2942.

Marconi, S., Castiglione, G., Turano, E., Bissolotti, G., Angiari, S., Farinazzo, A., ... Bonetti, B. (2012). Human adipose-derived mesenchymal stem cells systemically injected promote peripheral nerve regeneration in the mouse model of sciatic crush. Tissue Engineering Part A, 18, 1264-1272.

Martino, M. M., Briquez, P. S., Ranga, A., Lutolf, M. P., \& Hubbell, J. A. (2013). Heparin-binding domain of fibrin(ogen) binds growth factors and promotes tissue repair when incorporated within a synthetic matrix. Proceedings of the National Academy of Sciences, 110, 4563-4568.

Minev, I. R., Moshayedi, P., Fawcett, J. W., \& Lacour, S. P. (2013). Interaction of glia with a compliant, microstructured silicone surface. Acta Biomaterialia, 9, 6936-6942.

Minev, I. R., Musienko, P., Hirsch, A., Barraud, Q., Wenger, N., Moraud, E. M., ... Lacour, S. P. (2015). Electronic dura mater for long-term multimodal neural interfaces. Science, 347(6218), 159-163.

Musick, K. M., Rigosa, J., Narasimhan, S., Wurth, S., Capogrosso, M., Chew, D. J., ... Lacour, S. P. (2015). Chronic multichannel neural recordings from soft regenerative microchannel electrodes during gait. Scientific Reports, 5, 14363.

Pittier, R., Sauthier, F., Hubbell, J. A., \& Hall, H. (2005). Neurite extension and in vitro myelination within three-dimensional modified fibrin matrices. Journal of Neurobiology, 63, 1-14.

Rao, S. S., \& Winter, J. O. (2009). Adhesion molecule-modified biomaterials for neural tissue engineering. Nature Clinical Practice Neurology, 2, 6.

Reichenberger, M. A., Mueller, W., Hartmann, J., Diehm, Y., Lass, U., Koellensperger, E., ... Fischer, S. (2016). ADSCs in a fibrin matrix enhance nerve regeneration after epineural suturing in a rat model. Microsurgery, 36, 491-500.

Reid, A. J., Sun, M., Wiberg, M., Downes, S., Terenghi, G., \& Kingham, P. J. (2011). Nerve repair with adipose-derived stem cells protects dorsal root ganglia neurons from apoptosis. The Journal of Neuroscience, 199, 515-522.

Sacchi, V., Mittermayr, R., Hartinger, J., Martino, M. M., Lorentz, K. M., Wolbank, S., ... Banfi, A. (2014). Long-lasting fibrin matrices ensure stable and functional angiogenesis by highly tunable, sustained delivery of recombinant VEGF164. Proceedings of the National Academy of Sciences, 111, 6952-6957.

Saheb-Al-Zamani, M., Yan, Y., Farber, S. J., Hunter, D. A., Newton, P., Wood, M. D., ... Mackinnon, S. E. (2013). Limited regeneration in long acellular nerve allografts is associated with increased Schwann cell senescence. Experimental Neurology, 247, 165-177.

Santiago, L. Y., Clavijo-Alvarez, J., Brayfield, C., Rubin, J. P., \& Marra, K. G. (2009). Delivery of adipose-derived precursor cells for peripheral nerve repair. Cell Transplantation, 18, 145-158.

Schneider, C. A., Rasband, W. S., \& Eliceiri, K. W. (2012). NIH image to ImageJ: 25 years of image analysis. Nature Methods, 9, 671-675.

Seidel, M., Borczynska, A., Rozwadowska, N., \& Kurpisz, M. (2009). Cellbased therapy for heart failure: Skeletal myoblasts. Cell Transplantation, 18, 695-707.

Sowa, Y., Imura, T., Numajiri, T., Nishino, K., \& Fushiki, S. (2012). Adiposederived stem cells produce factors enhancing peripheral nerve regeneration: Influence of age and anatomic site of origin. Stem Cells and Development, 21, 1852-1862.

Srinivasan, A., Tahilramani, M., Bentley, J. T., Gore, R. K., Millard, D. C., Mukhatyar, V. J., ... Bellamkonda, R. V. (2015). Microchannel-based regenerative scaffold for chronic peripheral nerve interfacing in amputees. Biomaterials, 41, 151-165.

Srinivasan, A., Tipton, J., Tahilramani, M., Kharbouch, A., Gaupp, E., Song, C., ... Bellamkonda, R. V. (2016). A regenerative microchannel device for recording multiple single-unit action potentials in awake, ambulatory animals. European Journal of Neuroscience, 43(3), 474-485.

Suganuma, S., Tada, K., Hayashi, K., Takeuchi, A., Sugimoto, N., Ikeda, K., \& Tsuchiya, H. (2013). Uncultured adipose-derived regenerative cells promote peripheral nerve regeneration. Journal of Orthopaedic Science, 18 , 145-151.

di Summa, P. G., Kalbermatten, D. F., Pralong, E., Raffoul, W., Kingham, P. J., \& Terenghi, G. (2011). Long-term in vivo regeneration of peripheral nerves through bioengineered nerve grafts. The Journal of Neuroscience, 181, 278-291.

di Summa, P. G., Kalbermatten, D. F., Raffoul, W., Terenghi, G., \& Kingham, P. J. (2013). Extracellular matrix molecules enhance the neurotrophic effect of Schwann cell-like differentiated adipose-derived stem cells 
and increase cell survival under stress conditions. Tissue Engineering Part A, 19, 368-379.

Tashiro, K., Sephel, G. C., Weeks, B., Sasaki, M., Martin, G. R., Kleinman, H. K., \& Yamada, Y. (1989). A synthetic peptide containing the IKVAV sequence from the $A$ chain of laminin mediates cell attachment, migration, and neurite outgrowth. The Journal of Biological Chemistry, 264, 16174-16182.

Tohill, M., \& Terenghi, G. (2004). Stem-cell plasticity and therapy for injuries of the peripheral nervous system. Biotechnology and Applied Biochemistry, 40, 17-24.

Tortelli, F., Pisano, M., Briquez, P. S., Martino, M. M., \& Hubbell, J. A. (2013). Fibronectin binding modulates CXCL11 activity and facilitates wound healing. PloS One, 8, e79610.

Wang, S., Cai, Q., Hou, J., Bei, J., Zhang, T., Yang, J., \& Wan, Y. (2003). Acceleration effect of basic fibroblast growth factor on the regeneration of peripheral nerve through a 15-mm gap. Journal of Biomedical Materials Research Part A, 66, 522-531.

Wei, X., Zhao, L., Zhong, J., Gu, H., Feng, D., Johnstone, B. H., ... Du, Y. (2009). Adipose stromal cells-secreted neuroprotective media against neuronal apoptosis. Neuroscience Letters, 462, 76-79.
Yu, X. J., \& Bellamkonda, R. V. (2003). Tissue-engineered scaffolds are effective alternatives to autografts for bridging peripheral nerve gaps. Tissue Engineering, 9, 421-430.

Zhao, L., Wei, X., Ma, Z., Feng, D., Tu, P., Johnstone, B. H., ... Du, Y. (2009). Adipose stromal cells-conditional medium protected glutamate-induced CGNs neuronal death by BDNF. Neuroscience Letters, 452, 238-240.

Zuk, P. A., Zhu, M., Ashjian, P., De Ugarte, D. A., Huang, J. I., Mizuno, H., ... Hedrick, M. H. (2002). Human adipose tissue is a source of multipotent stem cells. Molecular Biology of the Cell, 13, 4279-4295.

How to cite this article: de Luca AC, Fonta CM, Raffoul W, di Summa PG, and Lacour SP. In vitro evaluation of gel-encapsulated adipose derived stem cells: Biochemical cues for in vivo peripheral nerve repair. J Tissue Eng Regen Med. 2017;1-11 https://doi.org/10.1002/term.2486 